\title{
Sodium Metasilicate Cemented Analogue Material and Its Mechanical Properties
}

\author{
Songlin Yue,, ${ }^{1,2}$ Yanyu Qiu, ${ }^{1}$ Pengxian Fan, ${ }^{1}$ Pin Zhang, ${ }^{3}$ and Ning Zhang ${ }^{1,4}$ \\ ${ }^{1}$ State Key Laboratory for Disaster Prevention \& Mitigation of Explosion \& Impact, PLA University of Science and Technology, \\ Nanjing 210007, China \\ ${ }^{2}$ State Key Laboratory of Coal Resources and Safe Mining, China University of Mining \& Technology, Xuzhou, Jiangsu 221008, China \\ ${ }^{3}$ National Key Laboratory on Electromagnetic Environment and Electrooptical Engineering, PLA University of Science \& Technology, \\ Nanjing 210007, China \\ ${ }^{4}$ College of Science, Nanjing University of Science and Technology, Nanjing 210007, China
}

Correspondence should be addressed to Pengxian Fan; fan-px@139.com and Pin Zhang; pinzhangthree@sina.com

Received 8 March 2016; Revised 27 June 2016; Accepted 4 July 2016

Academic Editor: Jun Liu

Copyright (C) 2016 Songlin Yue et al. This is an open access article distributed under the Creative Commons Attribution License, which permits unrestricted use, distribution, and reproduction in any medium, provided the original work is properly cited.

\begin{abstract}
Analogue material with appropriate properties is of great importance to the reliability of geomechanical model test, which is one of the mostly used approaches in field of geotechnical research. In this paper, a new type of analogue material is developed, which is composed of coarse aggregate (quartz sand and/or barite sand), fine aggregate (barite powder), and cementitious material (anhydrous sodium silicate). The components of each raw material are the key influencing factors, which significantly affect the physical and mechanical parameters of analogue materials. In order to establish the relationship between parameters and factors, the material properties including density, Young's modulus, uniaxial compressive strength, and tensile strength were investigated by a series of orthogonal experiments with hundreds of samples. By orthogonal regression analysis, the regression equations of each parameter were obtained based on experimental data, which can predict the properties of the developed analogue materials according to proportions. The experiments and applications indicate that sodium metasilicate cemented analogue material is a type of low-strength and low-modulus material with designable density, which is insensitive to humidity and temperature and satisfies mechanical scaling criteria for weak rock or soft geological materials. Moreover, the developed material can be easily cast into structures with complex geometry shapes and simulate the deformation and failure processes of prototype rocks.
\end{abstract}

\section{Introduction}

Geomechanical model test is one of the most widely used approaches in field of geotechnical and geology research $[1,2]$. The accuracy and reliability of geomechanical model tests depend on the similarity of physical process, which involves geometric, boundary, initial conditions and scale criterion of physical material parameters [3-5]. Use of analogue material is the key of ensuring similarity between model and prototype $[6,7]$. Thus, the preparation of analogue materials is a fundamental problem of geomechanical model tests.

Analogue materials research started in Europe. In the 1960s, Fumagalli [8] pioneered techniques for geomechanical model tests in the Experimental Institute for Models and Structures (ISMES). They developed analogue materials which used gypsum as a binder and lead oxide powder and bentonite clay as filler materials. Then, Stimpson [9] gave a detailed review of various constituents that have been used in the past and group the materials as cemented and noncemented, with plaster and ordinary Portland cement being the most common cementing agents. In order to simulate coal rock, Burgert and Lippmann [10] developed a new analogue material which was made by adding a kind of hardener into epoxy resin. Indraratna [11] presented a synthetic material to simulate soft sedimentary rocks and it was constituted with gypsum cement, fine sand, and water. Glushinkhin et al. [12] developed a series of analogue materials and applied them in model tests of zonal disintegration in deep rock masses, 


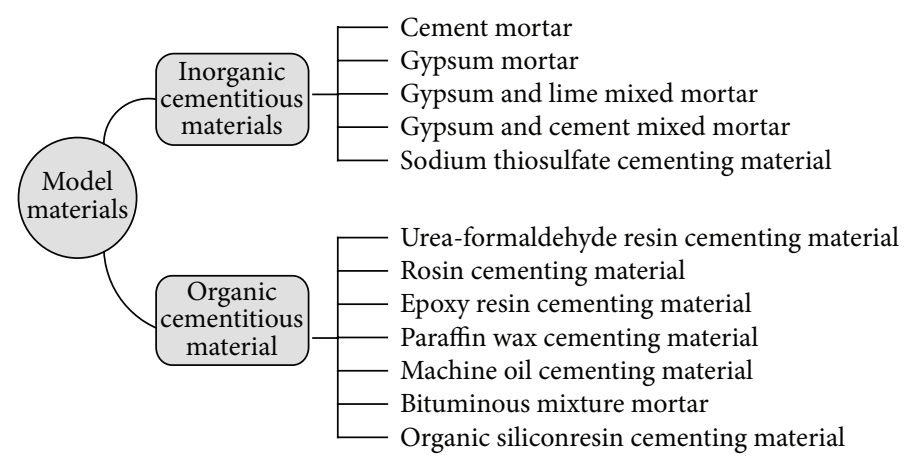

FIGURE 1: Classification of analogue materials for rock [8].

mining disturbances, and so on. Dykeman and Valsangkar [13] presented results of centrifuge modelling of socketed caissons in a weak model rock made of cement, sand, bentonite, and water. Another kind of analogue material (NIOS) was developed by Li et al. [14], which contained magnetite powder, sand, cement or gypsum, water (as a mixing agent), and an additive. Dunham et al. [15] performed series of centrifuge tests by using a model rock made from a mixture of sand, bentonite, cement, and water. Chen and Bai [16] developed a type of analogue material to simulate a rockburst by using a mixture of quartz sand, gypsum, cement, water, glycerine, gelatin, and so forth. This kind of material has a low density and low Poisson's ratio. Zhang et al. [17] developed analogue material by using iron ore powder, barite powder, and quartz sand as the aggregates, rosin and alcohol as the cementing agent, and gypsum powder as a conditioning agent. The material has the advantages of a high density, a wide range of parameter variation, and a stable performance: it can be used to simulate most rock masses between soft rock and hard rock. Imre et al. [18] provided a recipe of a synthetic cemented sand, together with a comprehensive characterisation for its mechanical properties. The material was named ETH analogue material for rock (ETHAR).

Generally, analogue materials can be divided into two types, organic cementitious materials and inorganic cementitious materials, and can be further subdivided into more than a dozen subclasses, shown in Figure 1 [8]. Most of organic cementitious materials are shaped by compressing, which cannot cast into large dimensions or structural mould. Inorganic cementitious materials can be easily shaped without external pressure, but most of them have properties with low density and high strength. Specifically, cement mortar and sodium thiosulfate cementing material are casting materials, but they always have high strength. Gypsum mortar and gypsum and cement mixed mortar are sensitive to humidity, which limit their application.

According to Indraratna's suggestions [11], excellent analogue material should meet the following specifications: (a) satisfy mechanical scaling criteria; (b) be mouldable or can be cast into mould; (c) be insensitive to heat and humidity; (d) be economical and environmental friendly; (e) have a short curing time.
Geomechanical model test is built on strict similarity law, which should satisfy simulated condition based on equilibrium equations, geometric equations, physical equations, boundary conditions, and displacement conditions $[19,20]$. And the single valued similar conditions should meet the following similarity criteria [21, 22]:

$$
\begin{aligned}
& \alpha_{\sigma}=\alpha_{\rho g} \alpha_{L} \\
& \alpha_{\delta}=\alpha_{E} \alpha_{L} \\
& \alpha_{\sigma}=\alpha_{E} \alpha_{\varepsilon} \\
& \alpha_{t}=\sqrt{\alpha_{L}} \\
& \alpha_{\varepsilon}=1, \\
& \alpha_{\varphi}=1, \\
& \alpha_{\mu}=1,
\end{aligned}
$$

where the operator $\alpha$ is the notation of the ratio/scale of prototype materials' parameters to analogue materials'. According to similarity criteria above, in the process of preparing analogue materials, bulk density should keep constant, but Young's modulus, stress, and strength should be scaled as geometric scale [23]. Therefore, analogue materials always have the characteristics of high density, low strength, and Young's modulus.

Although scholars have tried many material preparation methods, most of them cannot satisfy mechanical scaling criteria for weak rock or soft geological materials, or some incur high cost, are complex, and offer low controllability of mechanical properties. Therefore, a new type of analogue material that meets excellent analogue material's specifications should be developed.

In this paper, we present a type of material with high density, low strength and Young's modulus, easy mouldability, and good stability, which can simulate weak rock or soft geological materials. In order to investigate the properties of the proposed material, orthogonal experiments are conducted and relationships between the physicomechanical properties 


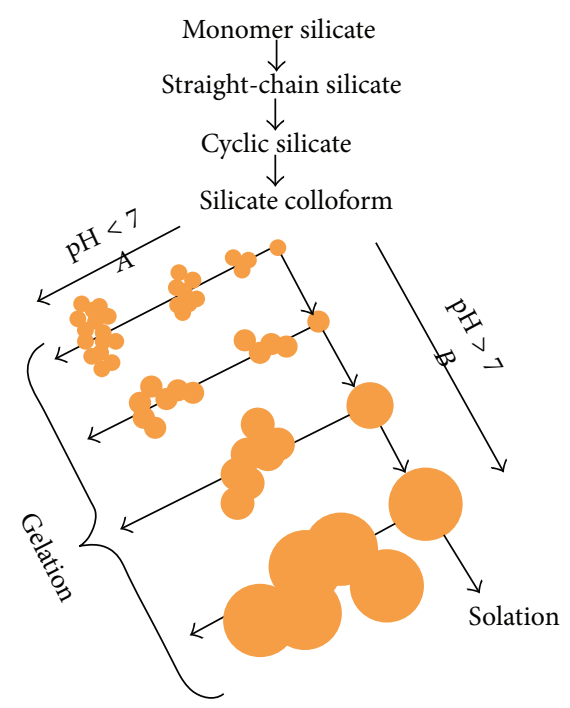

FIGURE 2: Growth of colloidal particle and gelatinization [22].

of the analogue material and influencing factors are established by regression analysis.

\section{The Curing Mechanism of Anhydrous Sodium Silicate}

Sodium silicate sand is a type of material used to make moulds and is widely used in the casting industry. However, the silicate content of sodium silicate cannot be accurately controlled and lead to a wide variation range of material properties. Therefore, sodium metasilicate solution is used here to allow control of the silicate content.

Sodium metasilicate often contains water of crystallisation and thus forms sodium metasilicate pentahydrate, or sodium metasilicate nonahydrate, but all these compounds have a low water solubility. Therefore, anhydrous sodium silicate powder was used as the raw material, because of its high water solubility. Sodium metasilicate can react with $\mathrm{CO}_{2}$ and harden, but the hardening process is slow, and what is worse is that a hardened layer will form on the surface and prevent the full reaction of the inner parts. Therefore, sodium fluorosilicate was used as a curing agent to accelerate the process.

The chemical formula for sodium fluorosilicate is $\mathrm{Na}_{2} \mathrm{SiF}_{6}$ : which has a low water solubility and can be mixed with aggregate. Then it is stirred with the sodium metasilicate solution, after which it starts to harden. Specimens were made by casting the unset mixture into a mould and allowing it to harden.

The chemical reaction between sodium metasilicate and sodium fluorosilicate is

$$
2 \mathrm{Na}_{2} \mathrm{SiO}_{3}+\mathrm{Na}_{2} \mathrm{SiF}_{6}+6 \mathrm{H}_{2} \mathrm{O} \longrightarrow 6 \mathrm{NaF}+3 \mathrm{Si}(\mathrm{OH})_{4}
$$

There are two reaction products: $\mathrm{NaF}$ and $\mathrm{Si}(\mathrm{OH})_{4} \cdot \mathrm{NaF}$ will separate out from the solution. As shown in Figure 2, $\mathrm{Si}(\mathrm{OH})_{4}$ will gelate when the $\mathrm{pH}$ is less than 7 but will solate when the $\mathrm{pH}$ is alkaline.
TABLE 1: Levels of each factor.

\begin{tabular}{lcccc}
\hline Level & & \multicolumn{3}{c}{ Factor } \\
& $A$ & $B$ & $C$ & $D$ \\
\hline 1 & $40 \%$ & 0 & $1 \%$ & $1 / 4$ \\
2 & $50 \%$ & $1 / 3$ & $3 \%$ & $2 / 4$ \\
3 & $60 \%$ & $2 / 3$ & $5 \%$ & $3 / 4$ \\
4 & $70 \%$ & 1 & $7 \%$ & $4 / 4$ \\
\hline
\end{tabular}

TABLE 2: The proportions of raw materials of each group.

\begin{tabular}{lcccc}
\hline \multirow{2}{*}{ Number } & \multicolumn{4}{c}{ Factors } \\
\hline 1 & $A$ & $B$ & $C$ & $D$ \\
2 & $40 \%$ & 0 & $1 \%$ & $1 / 4$ \\
3 & $40 \%$ & $1 / 3$ & $3 \%$ & $2 / 4$ \\
4 & $40 \%$ & $2 / 3$ & $5 \%$ & $3 / 4$ \\
5 & $40 \%$ & 1 & $7 \%$ & $4 / 4$ \\
6 & $50 \%$ & 0 & $3 \%$ & $3 / 4$ \\
7 & $50 \%$ & $1 / 3$ & $1 \%$ & $4 / 4$ \\
8 & $50 \%$ & $2 / 3$ & $7 \%$ & $1 / 4$ \\
9 & $50 \%$ & 1 & $5 \%$ & $2 / 4$ \\
10 & $60 \%$ & 0 & $5 \%$ & $4 / 4$ \\
11 & $60 \%$ & $1 / 3$ & $7 \%$ & $3 / 4$ \\
12 & $60 \%$ & $2 / 3$ & $1 \%$ & $2 / 4$ \\
13 & $60 \%$ & 1 & $3 \%$ & $1 / 4$ \\
14 & $70 \%$ & 0 & $7 \%$ & $2 / 4$ \\
15 & $70 \%$ & $1 / 3$ & $5 \%$ & $1 / 4$ \\
16 & $70 \%$ & $2 / 3$ & $3 \%$ & $4 / 4$ \\
\hline
\end{tabular}

\section{Sample Preparation and Testing Programme}

3.1. The Proportion of Raw Materials. Analogue materials have different characteristics as a result of the differences in aggregates and cementitious materials used [24]. The proportion of raw materials can determine the parameters of analogue materials.

The raw materials were anhydrous sodium silicate, sodium fluorosilicate, quartz sand, barite sand, barite powder, and water. Anhydrous sodium silicate and sodium fluorosilicate are both pure granular materials; the sizes of quartz and barite and sand grains were 0.6 to $1.18 \mathrm{~mm}$; and the sizes of the barite powder grains were 0.06 to $0.1 \mathrm{~mm}$.

Therefore, material proportion can be determined by four coefficients: $A$ (the proportion of fine powder to aggregates), $B$ (the proportion of barite sand to coarse aggregate), $C$ (the mass ratio of anhydrous sodium silicate to aggregate), and $D$ (the mass ratio of sodium fluorosilicate to anhydrous sodium silicate). According to the orthogonal experimental method, tests can be distributed so as to clarify the relationship between experimental conditions and experimental results. As shown in Tables 1 and 2, the paper put forward an orthogonal experimental design, in which the level distributions of each factor and the proportions of raw materials of each group are listed. 

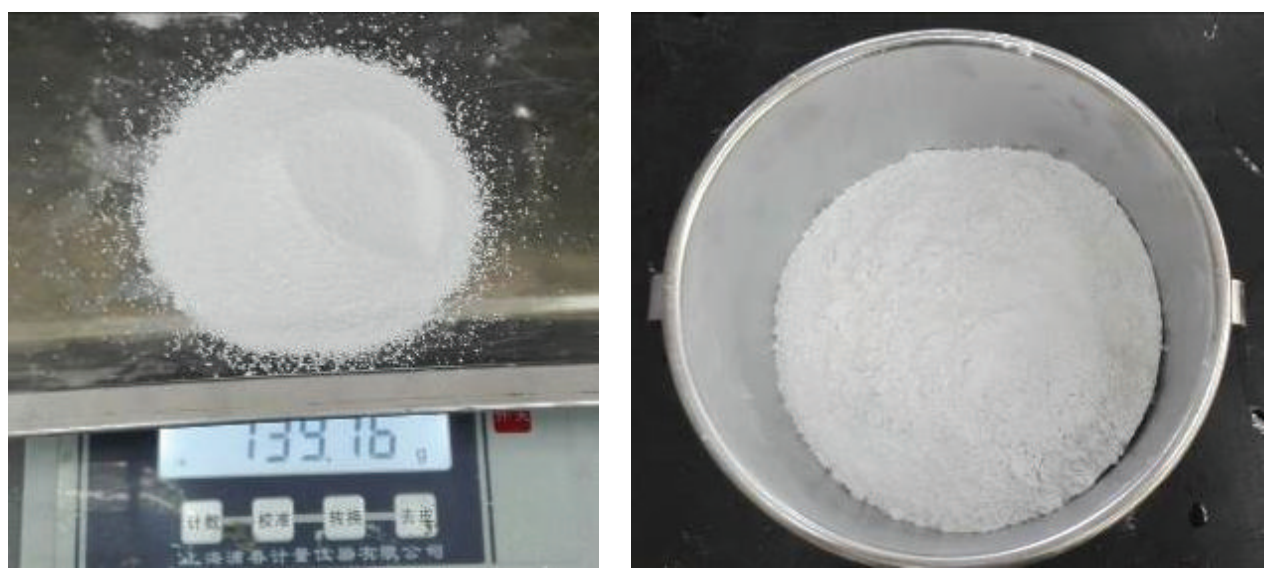

FIGURE 3: Weighing.
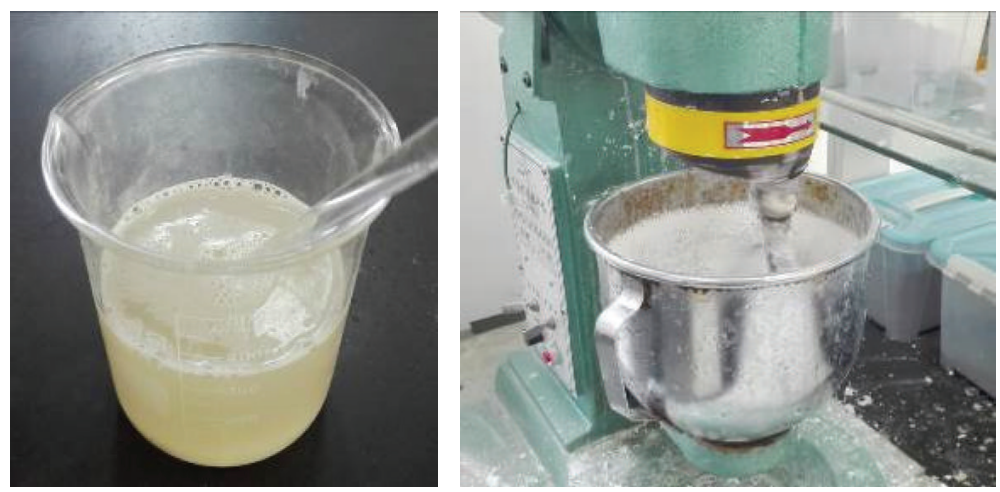

Figure 4: Agitation.

A four-factor, four-level test scheme was designed according to the orthogonal table $L_{16}\left(4^{5}\right)$, as shown in Table 2.

According to Tables 1 and 2, the proportion of raw materials of each group was determined.

3.2. Sample Preparation Process. In order to ensure the repeatability of tests [25], 5 uniaxial compressive specimens and 5 Brazil split specimens were prepared for each group simultaneously, and all the specimens were put into a standard curing room at $20^{\circ} \mathrm{C}$ and a relative humidity of $90 \%$ after stripping. The process of sample preparation was shown in the following specifications:

(1) Weighing (shown in Figure 3): Raw materials were weighed according to the mix design proportions, and the anhydrous sodium silicate was added into water to form an aqueous solution.

(2) Agitation (shown in Figure 4): First, cast the aggregate and sodium fluorosilicate into the mixer, and keep it dry while mixing until completely combined $(3 \mathrm{~min}$ ). Then cast the sodium silicate solution slowly into the mixer, and keep stirring for 5 min until mixed evenly.

(3) Casting (shown in Figure 5): After mixing, cast the mixture slowly into the mould and then vibrate it to

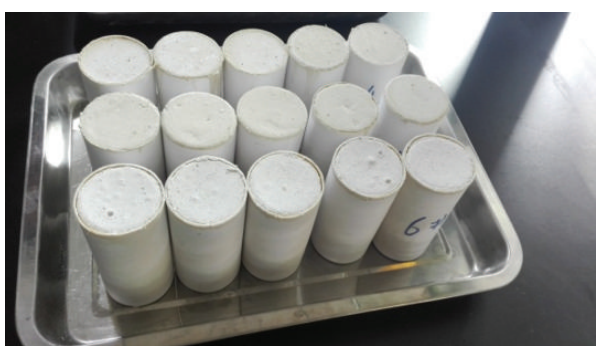

Figure 5: Casting.

prevent the generation of a honeycombed surface. The casting process must be completed within 20 minutes.

(4) Stripping (shown in Figure 6): In virtue of specimens that were in natural curing condition before stripping, the best time for stripping should be determined by the indoor environment. Due to the experimental time being longer, the temperature range was large. The stripping time was 5 days at 0 to $20^{\circ} \mathrm{C}$, and the stripping time is 3 days when the average temperature exceeds $20^{\circ} \mathrm{C}$.

(5) Curing: The curing serves several purposes: to accelerate the development of material strength and to 

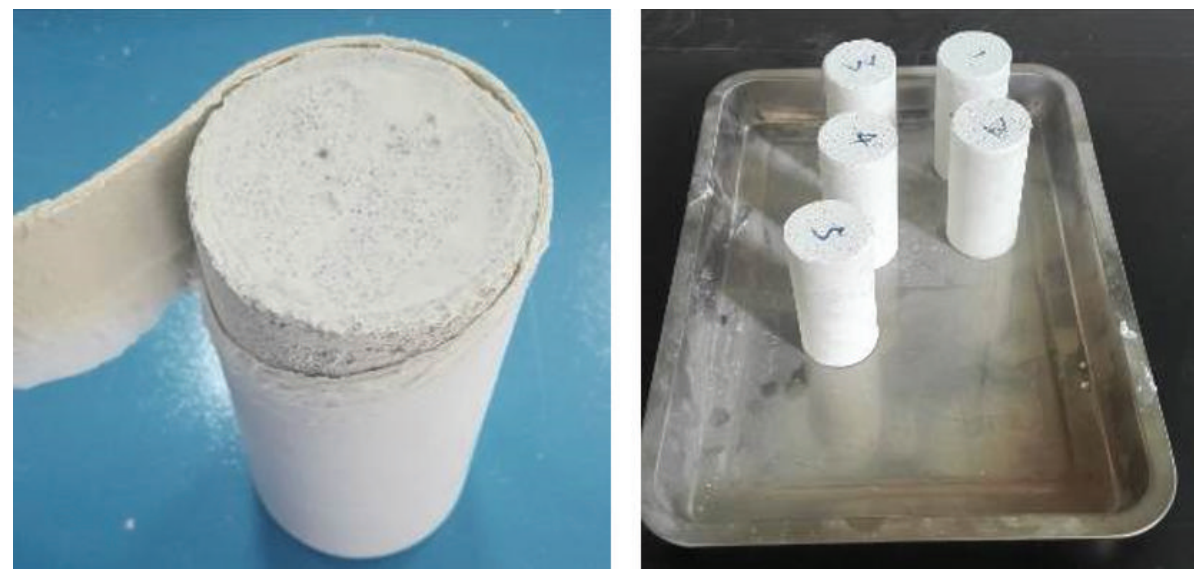

FIGURE 6: Stripping.

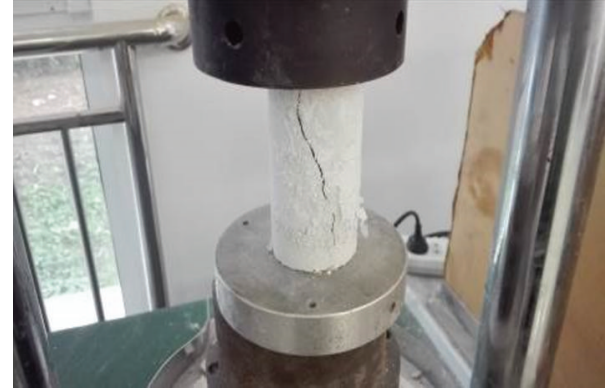

Figure 7: Uniaxial compressive strength test.

prevent cracking, shrinkage, and damage, which are caused by drying, temperature changes, and other natural factors. Specimens should be conserved under standard curing for 28 days.

3.3. Mechanical Testing Programme. Mechanical testing programme was carried out on pressing machine with the rate of displacement $0.02 \mathrm{~mm} / \mathrm{min}$.

In order to investigate the uniaxial compressive strength (UCS) (Figure 7) and Young's modulus of the new type of analogue materials, 16 groups of uniaxial compressive strength tests were carried out. There were 5 specimens for each group in uniaxial compressive strength test, but the effective data for each group may be less.

The stress-strain curves from Group 3 are shown in Figure 8 where the maxima are the uniaxial compressive strength (UCS).

Besides, the curves of uniaxial compressive stress and strain from Group 7 are shown in Figure 9. On the uniaxial compressive stress-strain curve, the elastic region was welldefined and Young's modulus could be found from the slope of the plot therein.

Young's modulus can be calculated as the following:

$$
E=\frac{\left(f_{c 1}-f_{c 2}\right)}{\left(\varepsilon_{1}-\varepsilon_{2}\right)}
$$

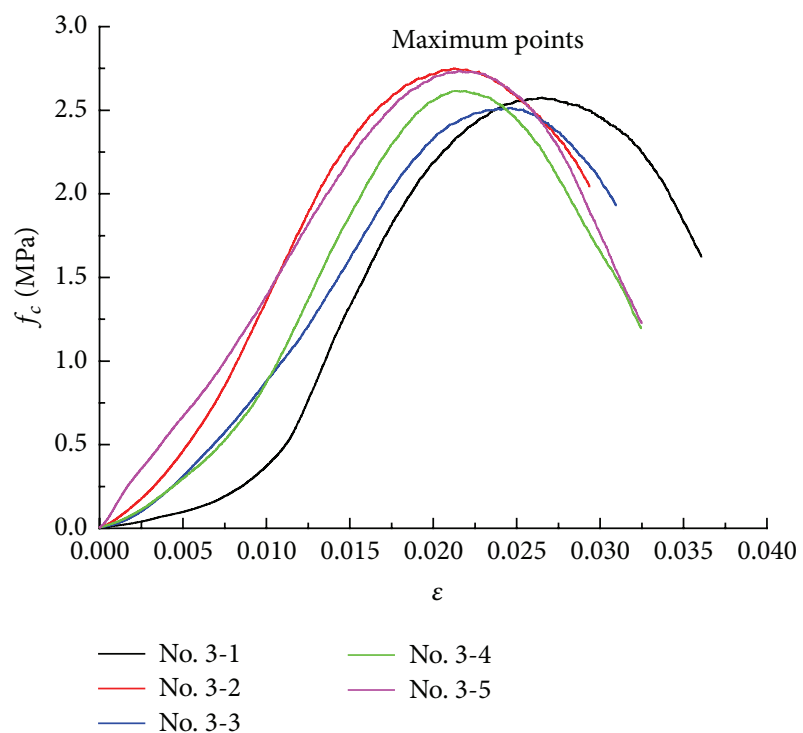

FIGURE 8: Uniaxial compressive stress-strain plots: Group 3.
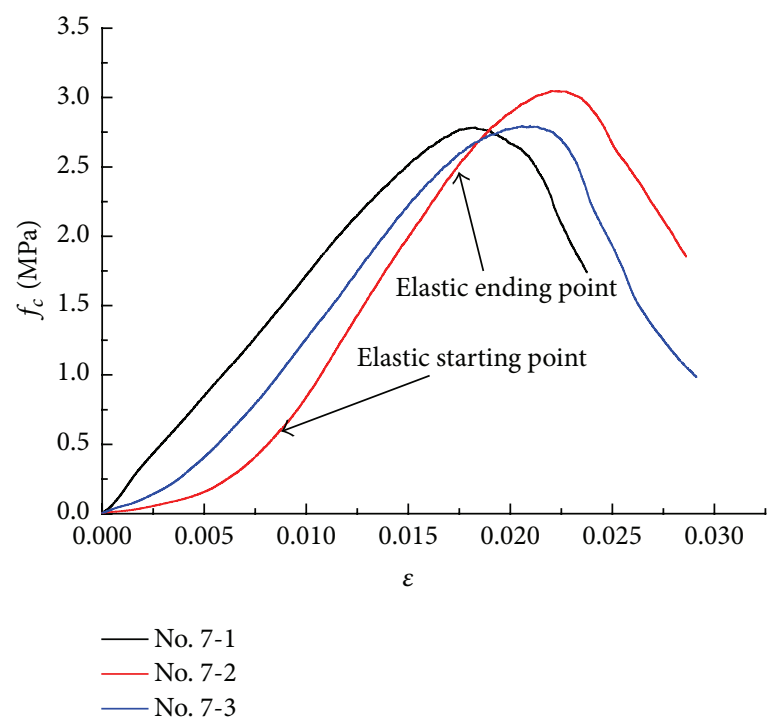

FIGURE 9: Uniaxial compressive stress-strain plots: Group 7. 


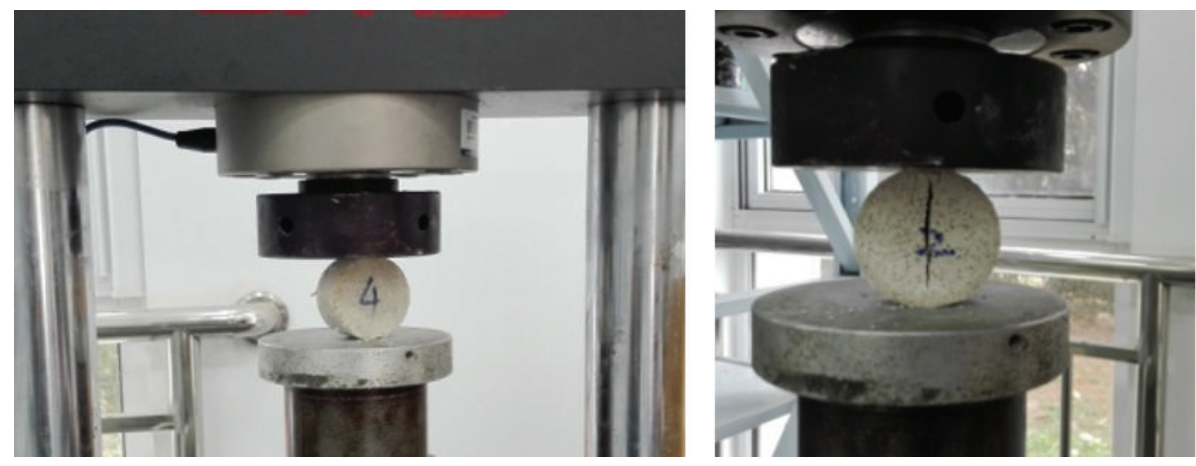

FIGURE 10: Flattened Brazilian disk test or tensile splitting test.

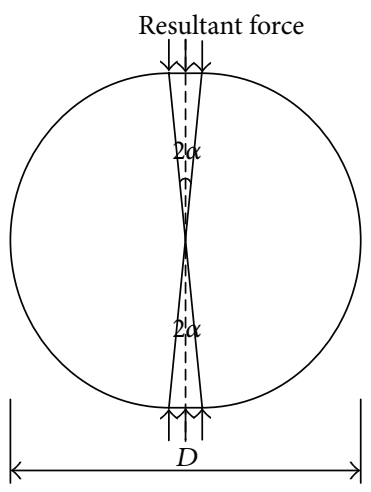

Figure 11: Flattened Brazilian disk specimen subjected to uniform diametral compression [26].

where $f_{c 1}, \varepsilon_{1}$ are, respectively, the stress and strain on elastic starting point and $f_{c 2}, \varepsilon_{2}$ are, respectively, the stress and strain on elastic ending point.

In order to investigate the tensile strength, 16 groups of flattened Brazilian disk tests (Figure 10) were carried out, and there were also 5 specimens for each group test.

According to Wang and Wu's suggestions [26], as shown in Figure 11, the tensile strength can be determined by the following formulae from a flattened Brazilian disk specimen:

$$
f_{t}=\frac{\left(1.92 P_{c}\right)}{(\pi D t)} \text {, }
$$

where $P_{c}$ is the critical load, $D$ is the diameter of the specimen, and $t$ is the thickness.

The load-time curves for the flattened Brazilian disk specimen used for the determination of rock tensile strength are shown in Figure 12 where the maxima denote critical tensile strengths.

\section{Results and Analysis}

4.1. Experimental Results. Experimental results including the data of density, Young's modulus, uniaxial compression strength, and Brazil splitting strength were listed in the Appendix.

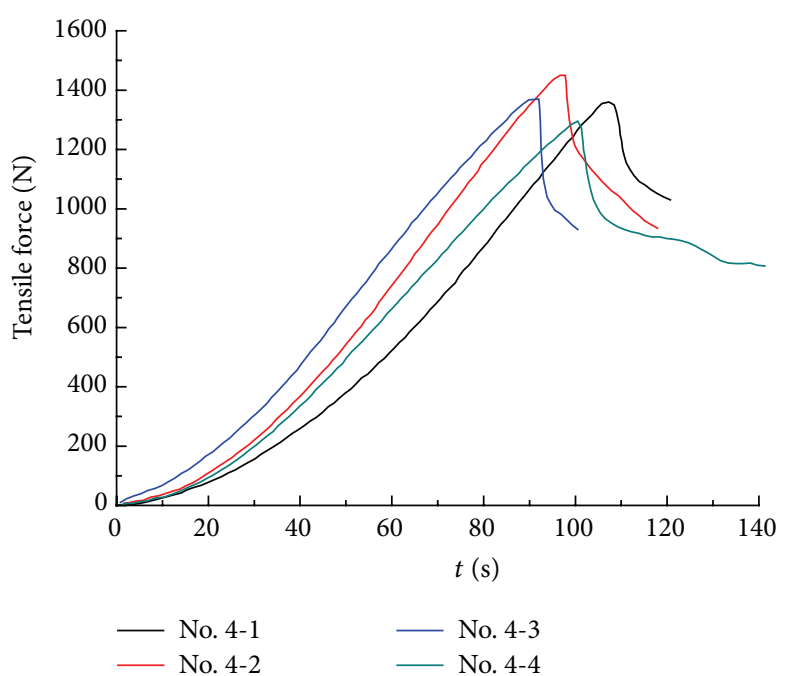

FIGURE 12: Load-time curve: Brazilian disk specimen used for the determination of rock tensile strength.

4.2. Analysis Method. The total effect function of all factors can be expressed as the sum of each factor effect:

$$
y=b_{0}+P(A)+P(B)+P(C)+\cdots,
$$

where $P(A), P(B), P(C), \ldots$ denote the effect of $A, B, C$, and so forth, respectively. The effect function of each factor can be expanded according to the orthogonal polynomial:

$$
\begin{aligned}
P(A)= & b_{1 a} \Phi_{1}(A)+b_{2 a} \Phi_{2}(A)+\cdots \\
& +b_{(n-1) a} \Phi_{(n-1)}(A) \\
= & b_{1 a} \lambda_{1 a} \Psi_{1}(A)+b_{2 a} \lambda_{2 a} \Psi_{2}(A)+\cdots \\
& +b_{(n-1) a} \lambda_{(n-1) a} \Psi_{(n-1)}(A), \\
P(B)= & b_{1 b} \Phi_{1}(B)+b_{2 b} \Phi_{2}(B)+\cdots+b_{(n-1) b} \Phi_{(n-1)}(B) \\
= & b_{1 b} \lambda_{1 b} \Psi_{1}(B)+b_{2 b} \lambda_{2 b} \Psi_{2}(B)+\cdots \\
& +b_{(n-1) b} \lambda_{(n-1) b} \Psi_{(n-1)}(B),
\end{aligned}
$$




$$
\begin{aligned}
P(C)= & b_{1 c} \Phi_{1}(C)+b_{2 c} \Phi_{2}(C)+\cdots+b_{(n-1) c} \Phi_{(n-1)}(C) \\
= & b_{1 c} \lambda_{1 c} \Psi_{1}(C)+b_{2 c} \lambda_{2 c} \Psi_{2}(C)+\cdots \\
& +b_{(n-1) c} \lambda_{(n-1) c} \Psi_{(n-1)}(C) .
\end{aligned}
$$

Regression coefficient $b_{k}$ and constant term $b_{0}$ can be calculated as follows:

$$
\begin{aligned}
& b_{k}=\frac{\sum_{t=1}^{n} \Phi_{i}\left(x_{t}\right) y_{t}}{r \sum_{t=1}^{n} \Phi_{i}^{2}\left(x_{t}\right)}=\frac{B_{i}}{r S_{i}}, \\
& b_{0}=\frac{1}{n r} \sum_{t=1}^{n} \sum_{j=1}^{r} y_{t j}=\frac{1}{n r} \sum_{t=1}^{n} y_{t},
\end{aligned}
$$

where $y_{t}=y_{t 1}+y_{t 1}+\cdots+y_{t 1}, t=1,2, \ldots, n$, and " $r$ " denotes repeat test times for the same factor at one level: the regression equation can then be established.

Using the following formula, the level of each factor is changed to a standard isometric point:

$$
\begin{aligned}
& A^{\prime}=\frac{(A-30 \%)}{(10 \%)} ; \\
& B^{\prime}=\frac{(B-(-1 / 3))}{(1 / 3)} ; \\
& C^{\prime}=\frac{(C-(-1 \%))}{(2 \%)} ; \\
& D^{\prime}=\frac{D}{(1 / 4)} .
\end{aligned}
$$

There were four level tests, and $n=4$; therefore each factor can be expanded to three terms. In this case, " $x$ " denotes the effect function of $A^{\prime}, B^{\prime}, C^{\prime}$, and $D^{\prime}$, in the orthogonal polynomials, and the regression equation can be expressed as

$$
\begin{aligned}
y= & b_{0}+b_{1 a} \lambda_{1 a} a+b_{2 a} \lambda_{2 a}\left[a^{2}-1.25\right] \\
& +b_{3 a} \lambda_{3 a}\left[a^{2}-2.05 a\right]+b_{1 b} \lambda_{1 b} b \\
& +b_{2 b} \lambda_{2 b}\left[b^{2}-1.25\right]+b_{3 b} \lambda_{3 b}\left[b^{2}-2.05 b\right] \\
& +b_{1 c} \lambda_{1 c} c+b_{2 c} \lambda_{2 c}\left[c^{2}-1.25\right] \\
& +b_{3 c} \lambda_{3 c}\left[c^{2}-2.05 c\right]+b_{1 d} \lambda_{1 d} d \\
& +b_{2 d} \lambda_{2 d}\left[d^{2}-1.25\right]+b_{3 d} \lambda_{3 d}\left[d^{2}-2.05 d\right],
\end{aligned}
$$

where

$$
\begin{aligned}
& a=\frac{(A-30 \%)}{(10 \%)}-2.5 ; \\
& b=\frac{(B-(-1 / 3))}{(1 / 3)}-2.5 ;
\end{aligned}
$$

$$
\begin{aligned}
& c=\frac{(C-(-1 \%))}{(2 \%)}-2.5 ; \\
& d=\frac{(D)}{(1 / 4)}-2.5 .
\end{aligned}
$$

The regression coefficients are calculated by using (7), in which $\Phi_{i}\left(x_{t}\right)$ and $\sum \Phi_{i}\left(x_{t}\right)^{2}$ values can be directly sought from the orthogonal polynomials table, and $b_{0}$ is calculated by use of (8).

To establish the optimal regression equation and the effect of the factors on the significance of the decision and to determine the significance of the regression coefficients, first of all, the sum of the squares variation of regression coefficients was evaluated, followed by an $F$-test. It is well known that

$$
l_{y y}=U+Q
$$

where $l_{y y}$ denotes the sum of the total squared variations, $U$ denotes the regression sum of the squares, and $Q$ denotes the residual sum of the squares. Also,

$$
\begin{aligned}
l_{y y} & =\sum_{t=1}^{n} \sum_{j=1}^{r} y_{t j}^{2}-\frac{1}{n r}\left(\sum_{t=1}^{n} \sum_{j=1}^{r} y_{t j}\right)^{2}, \\
U & =\sum_{i=1}^{k} b_{i} B_{i}=\sum_{i=1}^{k} b_{i}^{2}\left[r \sum_{t=1}^{n} \Phi_{i}^{2}\left(x_{t}\right)\right]=\sum_{i=1}^{k} b_{i}^{2} r S_{i}, \\
Q & =l_{y y}-U=\sum_{i=1}^{n} f_{i} S_{i}^{2} .
\end{aligned}
$$

$S_{i}^{2}$ is the variance of repeated measurements under the same conditions; $f_{i}$ is the number of degrees of freedom of the variance. The sum of the squares of the variation of the regression coefficients can be calculated as follows:

$$
S_{b_{i}}=b_{i} B_{i}=b_{i}^{2}\left(r S_{i}\right) .
$$

The numbers of degrees of freedom are

$$
\begin{aligned}
& f_{r}=n r-1 ; \\
& f_{u}=k ; \\
& f_{Q}=n r-k-1 ; \\
& f_{b_{i}}=1 ; \quad i=1,2, \ldots, k .
\end{aligned}
$$

4.2.1. Regression Analysis: Density. The density index results are listed in the Appendix (Tables 3 and 4). There are four levels $(n=4)$ for each factor and four groups of tests for each level. For each group, there are five effective values of the density index. The regression coefficients have been calculated based on formulas (7) and (8).

According to (14), the sum of the squares of the variations of the regression coefficients can be calculated (Table 4). 


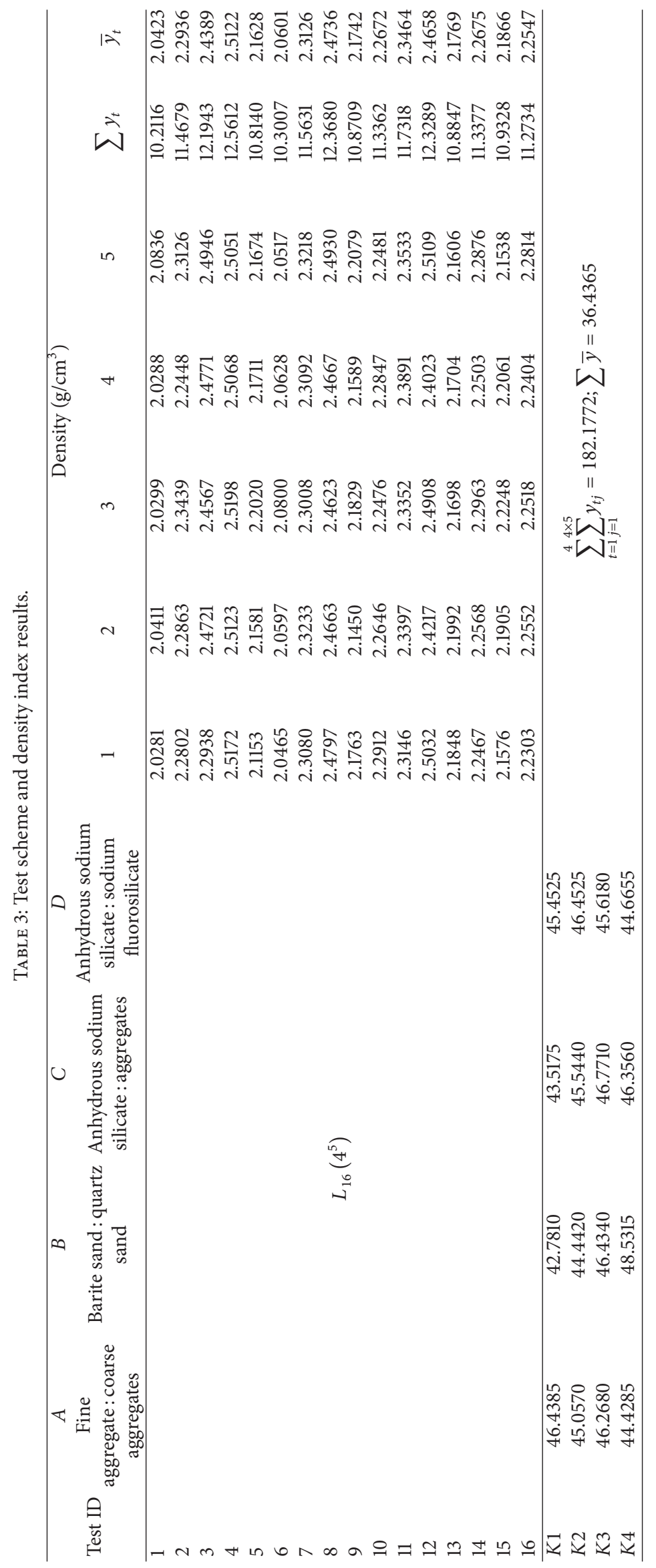


TABLE 4: Orthogonal polynomial regression analysis of variance: density index.

\begin{tabular}{|c|c|c|c|c|c|c|c|}
\hline $\begin{array}{l}\text { The source of } \\
\text { variance }\end{array}$ & $\begin{array}{l}\text { Square and } \\
\text { variation }\end{array}$ & $\begin{array}{l}\text { Degree of } \\
\text { freedom }\end{array}$ & $\begin{array}{l}\text { Variance } \\
\text { estimate }\end{array}$ & $F$-value & $F_{0.05}(1,69)$ & $\begin{array}{c}\text { Level of } \\
\text { significance }\end{array}$ & Remarks \\
\hline $\mathrm{b}_{1 a}$ & $5.8057 \times 10^{-2}$ & 1 & $5.8057 \times 10^{-2}$ & 28.70 & 4 & $* *$ & $A, 1$-order term \\
\hline$b_{2 a}$ & $2.6220 \times 10^{-3}$ & 1 & $2.6220 \times 10^{-3}$ & 1.30 & & & $A, 2$-order term \\
\hline$b_{3 a}$ & $7.9609 \times 10^{-2}$ & 1 & $7.9609 \times 10^{-2}$ & 39.36 & & $* *$ & $A, 3$-order term \\
\hline$b_{1 b}$ & $9.2578 \times 10^{-1}$ & 1 & $9.2578 \times 10^{-1}$ & 457.67 & & $* * * *$ & $B, 1$-order term \\
\hline$b_{2 b}$ & $2.3817 \times 10^{-3}$ & 1 & $2.3817 \times 10^{-3}$ & 1.18 & & & $B, 2$-order term \\
\hline$b_{1 c}$ & $2.3729 \times 10^{-1}$ & 1 & $2.3729 \times 10^{-1}$ & 117.31 & & $* * *$ & $C, 1$-order term \\
\hline$b_{2 c}$ & $7.4512 \times 10^{-2}$ & 1 & $7.4512 \times 10^{-2}$ & 36.84 & & $* *$ & $C, 2$-order term \\
\hline$b_{1 d}$ & $2.5528 \times 10^{-2}$ & 1 & $2.5528 \times 10^{-2}$ & 12.62 & & $*$ & $D, 1$-order term \\
\hline$b_{2 d}$ & $4.7653 \times 10^{-2}$ & 1 & $4.7653 \times 10^{-2}$ & 23.56 & & $* *$ & $D, 2$-order term \\
\hline$b_{3 d}$ & $7.3659 \times 10^{-3}$ & 1 & $7.3659 \times 10^{-3}$ & 3.64 & & & $D, 3$-order term \\
\hline Error & $1.3957 \times 10^{-2}$ & 69 & $2.0228 \times 10^{-3}$ & & & & \\
\hline
\end{tabular}

Besides, according to (13), other parameters can be calculated as follows:

$$
\begin{aligned}
l_{y y} & =1.600 ; \\
U & =1.463 ; \\
Q & =1.377 \times 10^{-1} \\
f_{r} & =79 ; \\
f_{U} & =12 ; \\
f_{Q} & =67 .
\end{aligned}
$$

The variance estimates of the error effect can be written as $\mathrm{Q} / f_{\mathrm{Q}}=2.055 \times 10^{-3}$. In Table $4, b_{3 b}$ and $b_{3 c}$ are the variance estimates of the regression coefficients which are both less than the error effects, and they can be merged therewith.

As shown in Table 4, the order of (decreasing) importance of the factors was $B, C, A$, and $D$. as

Regression analysis gives the optimal regression equation

$$
y_{d}=2.346+0.073 a+0.096 b+0.049 c-0.016 d \text {. }
$$

4.2.2. Regression Analysis: Young's Modulus. There were four levels $(n=4)$ for each factor and four groups of tests for each level. For each group of tests, there were three effectual pieces of data of the elasticity modulus index (see Appendix, Tables 5 and 6$)$.

To establish the optimal regression equation, it was important to judge the significance level of each factor, and to determine the significance of the regression coefficient, firstly, the regression coefficient of the variation of the squared sum was found, and then an $F$-test was conducted.

According to (13) and (14), the variation of the regression coefficients can be obtained (see Table 6):

$$
\begin{gathered}
l_{y y}=1.003 \times 10^{5} ; \\
U=9.439 \times 10^{4} ; \\
Q=5.938 \times 10^{3} ;
\end{gathered}
$$

$$
\begin{aligned}
& f_{r}=47 ; \\
& f_{u}=12 ; \\
& f_{Q}=35 .
\end{aligned}
$$

The results are listed in Table 6: the order of importance (decreasing) of the factors was $C, A, D$, and $B$.

Regression analysis gives the optimal regression equation as

$$
\begin{aligned}
y_{E}= & 49.980+12.391 a+6.430 b+27.928 c \\
& +15.503 d .
\end{aligned}
$$

4.2.3. Regression Analysis: UCS. The UCS results are listed in the Appendix (Tables 7 and 8).

To establish the optimal regression equation, it was important to judge the significance level of each factor, and to determine the significance of the regression coefficient, firstly, the regression coefficient of the variation of the squared sum was evaluated, and then an $F$-test was carried out.

According to (13) and (14), the variation of the regression coefficients can be obtained, as shown in Table 8:

$$
\begin{aligned}
l_{y y} & =97.877 ; \\
U & =9.300 \times 10^{1} ; \\
Q & =4.874 ; \\
f_{r} & =79 ; \\
f_{u} & =12 ; \\
f_{Q} & =67 .
\end{aligned}
$$

The results are listed in Table 8: for the compressive strength, the order of importance (decreasing) of the factors was $C, A, D$, and $B$.

Thus the optimal regression equation was

$$
y_{f_{c}}=0.639+0.277 a+0.482 b+1.015 c+0.519 d \text {. }
$$




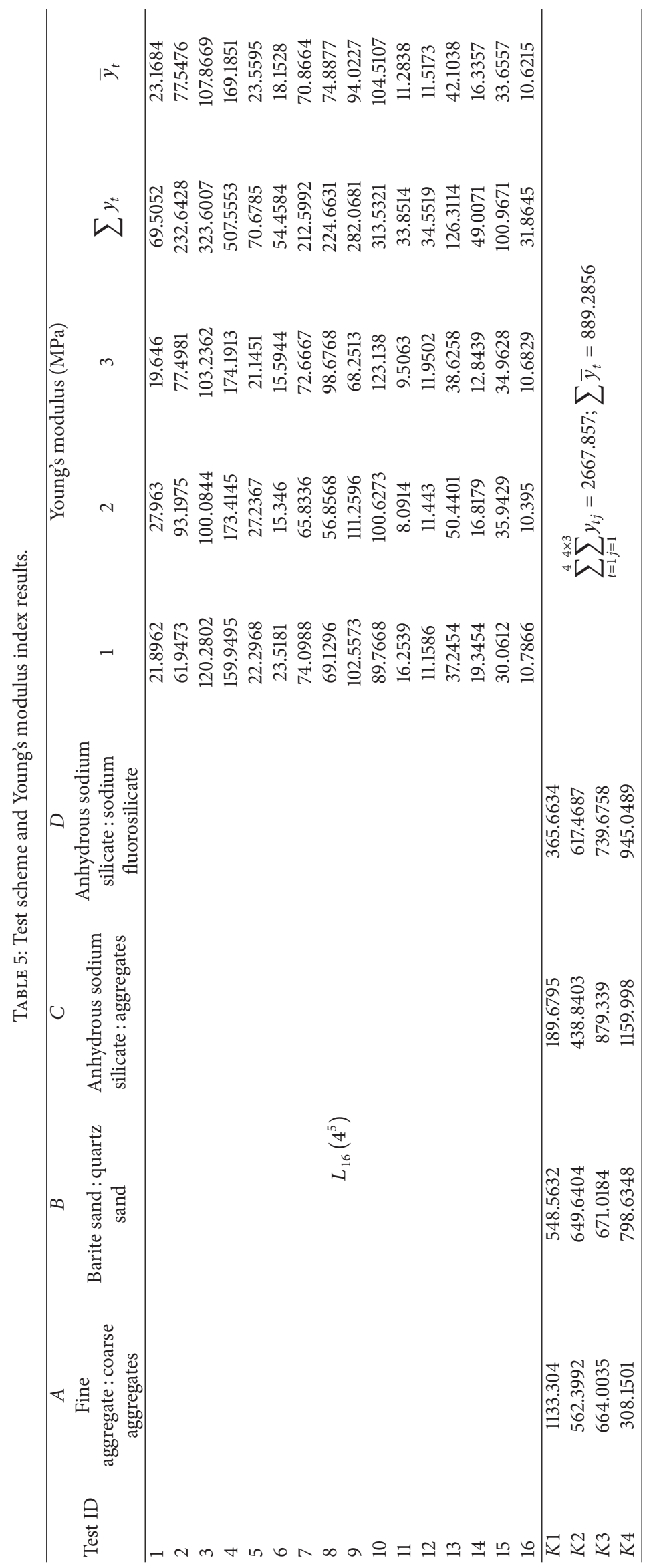


TABLE 6: Orthogonal polynomial regression analysis of variance: Young's modulus index.

\begin{tabular}{|c|c|c|c|c|c|c|c|}
\hline The source of variance & $\begin{array}{c}\text { Square and } \\
\text { variation }\end{array}$ & $\begin{array}{l}\text { Degree of } \\
\text { freedom }\end{array}$ & $\begin{array}{l}\text { Variance } \\
\text { estimate }\end{array}$ & $F$-value & $F_{0.05}(1,35)$ & $\begin{array}{c}\text { Level of } \\
\text { significance }\end{array}$ & Remarks \\
\hline$b_{1 a}$ & $2.3480 \times 10^{4}$ & 1 & $2.3480 \times 10^{4}$ & 138.39 & 4.13 & $* * * *$ & $\begin{array}{l}A, 1 \text {-order } \\
\text { term }\end{array}$ \\
\hline$b_{2 a}$ & $9.6348 \times 10^{2}$ & 1 & $9.6348 \times 10^{2}$ & 5.68 & & $*$ & $\begin{array}{c}A, 2 \text {-order } \\
\text { term }\end{array}$ \\
\hline$b_{3 a}$ & $5.3201 \times 10^{3}$ & 1 & $5.3201 \times 10^{3}$ & 31.36 & & $* * *$ & $\begin{array}{c}A, 3 \text {-order } \\
\text { term }\end{array}$ \\
\hline$b_{1 b}$ & $2.4806 \times 10^{3}$ & 1 & $2.4806 \times 10^{3}$ & 14.62 & & $* *$ & $\begin{array}{l}B, 1 \text {-order } \\
\text { term }\end{array}$ \\
\hline$b_{2 b}$ & $1.4674 \times 10^{1}$ & 1 & $1.4674 \times 10^{1}$ & 0.09 & & & $\begin{array}{c}B, 2 \text {-order } \\
\text { term }\end{array}$ \\
\hline$b_{3 b}$ & $1.4405 \times 10^{2}$ & 1 & $1.4405 \times 10^{2}$ & 0.85 & & & $\begin{array}{c}B, 3 \text {-order } \\
\text { term }\end{array}$ \\
\hline$b_{1 c}$ & $4.6801 \times 10^{4}$ & 1 & $4.6801 \times 10^{4}$ & 275.85 & & $* * * *$ & $\begin{array}{l}C, 1 \text {-order } \\
\text { term }\end{array}$ \\
\hline$b_{2 c}$ & $2.0670 \times 10^{1}$ & 1 & $2.0670 \times 10^{1}$ & 0.12 & & & $\begin{array}{c}C, 2 \text {-order } \\
\text { term }\end{array}$ \\
\hline$b_{3 c}$ & $5.1386 \times 10^{2}$ & 1 & $5.1386 \times 10^{2}$ & 3.03 & & & $\begin{array}{l}C, 3 \text {-order } \\
\text { term }\end{array}$ \\
\hline$b_{1 d}$ & $1.4421 \times 10^{4}$ & 1 & $1.4421 \times 10^{4}$ & 85.00 & & $* * * *$ & $\begin{array}{l}D, 1 \text {-order } \\
\text { term }\end{array}$ \\
\hline$b_{2 d}$ & $4.4916 \times 10^{1}$ & 1 & $4.4916 \times 10^{1}$ & 0.26 & & & $\begin{array}{l}D, 2 \text {-order } \\
\text { term }\end{array}$ \\
\hline$b_{3 d}$ & $1.8862 \times 10^{2}$ & 1 & $1.8862 \times 10^{2}$ & 1.11 & & & $\begin{array}{c}D, 3 \text {-order } \\
\text { term }\end{array}$ \\
\hline Error & $5.9382 \times 10^{3}$ & 35 & $1.6966 \times 10^{-2}$ & & & & \\
\hline
\end{tabular}

4.2.4. Regression Analysis: Tensile Strength. Tensile strengths and the regression coefficients are listed in the Appendix, Table 9.

To establish the optimal regression equation and the effect of the factors on the significance of the decision and to determine the significance of the regression coefficient, firstly, the regression coefficient of the variation of the squared sum was evaluated, and then an $F$-test was carried out. According to (13) and (14), the variation of the regression coefficients can be obtained, as shown in the Appendix, Table 10:

$$
\begin{aligned}
l_{y y} & =4.444 ; \\
U & =4.362 ; \\
Q & =8.163 \times 10^{-2} \\
f_{r} & =63 ; \\
f_{U} & =12 ; \\
f_{Q} & =51 .
\end{aligned}
$$

For the tensile strength, the order of importance (decreasing) was $C, A, D$, and $B$.

Thus the optimal regression equation was

$$
y_{f_{t}}=0.259+0.084 a+0.161 b+0.275 c+0.202 d
$$

\section{Application and Discussion}

This analogue material was applied in a model test that investigated the reinforcement effect on the upper part of the tunnel. In the model, the parameters of analogue material were determined by surrounding rock that was weak or soft rock in the prototype.

According to the method of analogue material preparation and the similarity laws for geomechanical models, analogue materials may be prepared by controlling the mechanical parameters of the materials.

Based on the scaling criterion, the scale factors for stress, length, and bulk density of a material have a determinate relationship [27]:

$$
\frac{\alpha_{\sigma}}{\alpha_{L} \alpha_{\gamma}}=1
$$

Given the scale factor for bulk densities is unity, the relationship can be transformed to [1]

$$
\alpha_{\sigma}=\alpha_{L}
$$

Therefore, the scales of mechanical parameters match the geometrical scale.

The geometric similarity ratio can be set based on the relevant scale of the model and the prototype: a value of 100 was chosen here. Therefore, the corresponding mechanical 


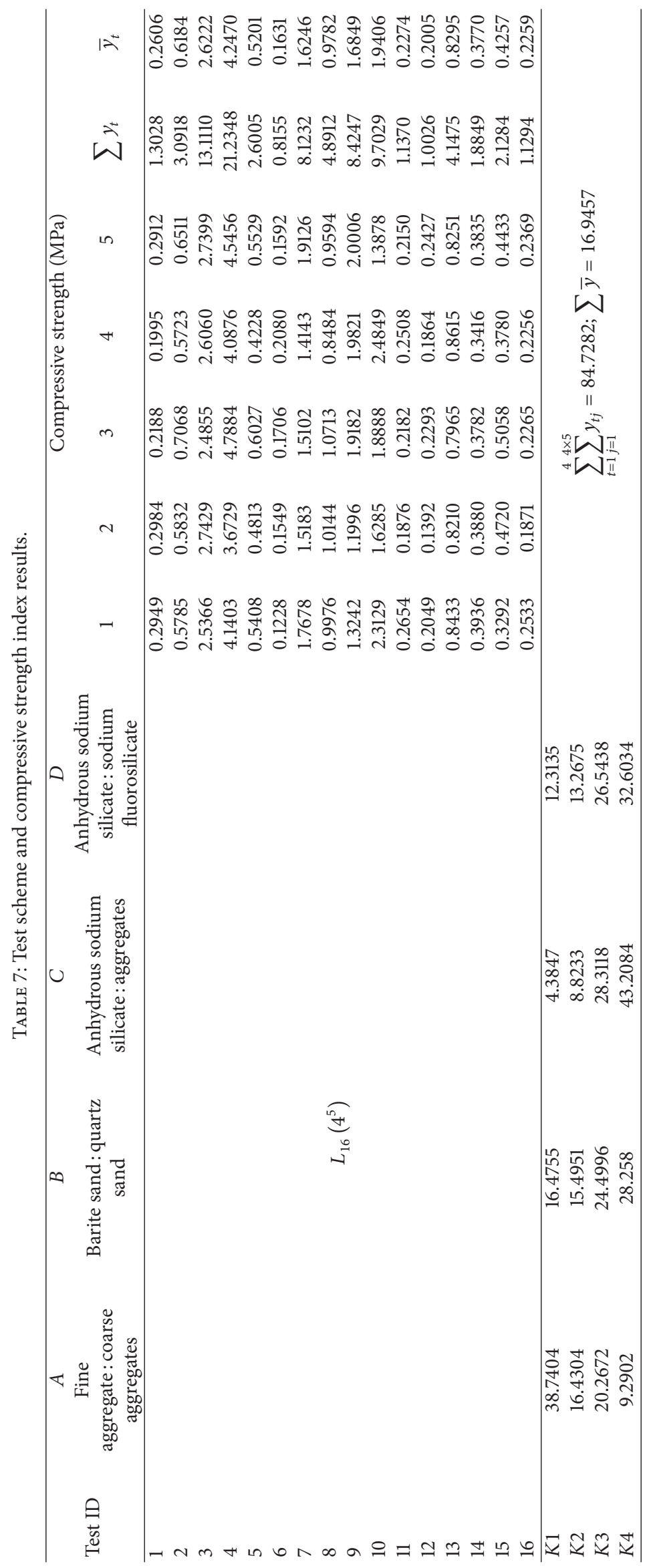


TABLE 8: Orthogonal polynomial regression analysis of variance: compressive strength index.

\begin{tabular}{|c|c|c|c|c|c|c|c|}
\hline The source of variance & $\begin{array}{l}\text { Square and } \\
\text { variation }\end{array}$ & $\begin{array}{l}\text { Degree of } \\
\text { freedom }\end{array}$ & $\begin{array}{l}\text { Variance } \\
\text { estimate }\end{array}$ & $F$-value & $F_{0.05}(1,69)$ & $\begin{array}{c}\text { Level of } \\
\text { significance }\end{array}$ & Remarks \\
\hline$b_{1 a}$ & $1.7856 \times 10^{1}$ & 1 & $1.7856 \times 10^{1}$ & 245.48 & 4 & $* * * *$ & $A, 1$-order term \\
\hline$b_{2 a}$ & $1.6055 \times 10^{0}$ & 1 & $1.6055 \times 10^{0}$ & 22.07 & & $* *$ & $A, 2$-order term \\
\hline$b_{3 a}$ & $4.1944 \times 10^{0}$ & 1 & $4.1944 \times 10^{0}$ & 57.66 & & $* *$ & $A, 3$-order term \\
\hline$b_{1 b}$ & $4.9177 \times 10^{0}$ & 1 & $4.9177 \times 10^{0}$ & 67.61 & & $* * *$ & $B, 1$-order term \\
\hline$b_{2 b}$ & $2.8070 \times 10^{-1}$ & 1 & $2.8070 \times 10^{-1}$ & 3.86 & & & $B, 2$-order term \\
\hline$b_{3 b}$ & $5.7996 \times 10^{-1}$ & 1 & $5.7996 \times 10^{-1}$ & 7.97 & & * & $B, 3$-order term \\
\hline$b_{1 c}$ & $4.6213 \times 10^{1}$ & 1 & $4.6213 \times 10^{1}$ & 635.31 & & $* * * *$ & $C, 1$-order term \\
\hline$b_{2 c}$ & $1.3671 \times 10$ & 1 & $1.3671 \times 10$ & 18.79 & & $* *$ & $C, 2$-order term \\
\hline$b_{3 c}$ & $9.6450 \times 10^{-1}$ & 1 & $9.6450 \times 10^{-1}$ & 13.26 & & $* *$ & $C, 3$-order term \\
\hline$b_{1 d}$ & $1.3744 \times 10^{1}$ & 1 & $1.3744 \times 10^{1}$ & 188.95 & & $* * * *$ & $D, 1$-order term \\
\hline$b_{2 d}$ & $3.2584 \times 10^{-1}$ & 1 & $3.2584 \times 10^{-1}$ & 4.48 & & $*$ & $D, 2$-order term \\
\hline$b_{3 d}$ & $9.5443 \times 10^{-1}$ & 1 & $9.5443 \times 10^{-1}$ & 13.12 & & $*$ & $D, 3$-order term \\
\hline Error & $4.8736 \times 10^{0}$ & 67 & $7.2740 \times 10^{-2}$ & & & & \\
\hline
\end{tabular}

parameters of the model or analogue materials can be calculated according to the similarity law and the parameters of the prototype material.

Then the range of each mechanical parameter of the objective material was calculated (Table 11). According to the regression equations, the raw material configuration to meet the mechanical index requirements was calculated.

According to the raw material configuration (Table 12), two kinds of materials were made within the property ranges listed in Table 11. Therefore, both materials were able to demonstrate the accuracy of the proposed method and its results.

Subsequently, number 2 analogue material was applied in a geomechanical model test which investigated the antistrike property of reinforcement layer on the top of tunnel. Some photographs of the specimens made of number 2 analogue material have been shown in Figure 13; specifically, there are scaling tunnel models with or without reinforcement layers. Both of the two kinds of scaling tunnel models have been subjected to the same impact loads provided by a drop hammer test machine. It is obvious that reinforcement layers can improve the medium resistance on the top of tunnels.

Since it is an example of our material used in application, more details and data of the scaling tunnel model tests are not convenient to disclose.

Generally, the properties of the developed analogue materials can be predicted according to the proportions. The experiments and applications indicate that it is a type of excellent analogue material which satisfies mechanical scaling criteria for weak rock or soft geological materials, and it will have broad application prospects.

\section{Conclusions}

(1) A new type of analogue material is developed, which is composed of coarse aggregate (quartz sand and/or barite sand), fine aggregate (barite powder), and cementitious material (anhydrous sodium silicate). It is a type of low-strength and low-modulus material with designable density, which is insensitive to humidity and temperature and satisfies mechanical scaling criteria for weak rock or soft geological materials.

(2) In order to establish the relationship between parameters and factors, the material properties including density, Young's modulus, uniaxial compressive strength, and tensile strength were investigated by a series of orthogonal experiments with hundreds of samples. According to the orthogonal experimental method, a four-factor, four-level test scheme is designed for the new material according to the orthogonal table $L_{16}\left(4^{5}\right)$.

(3) The relationship between parameters and factors was obtained. For the density index, the most important factor is $B$ (the proportion of barite sand to coarse aggregate), followed by $C$ (the mass ratio of anhydrous sodium silicate to aggregate), and $A$ (the proportion of fine powder to aggregates), and the effects of $D$ (the mass ratio of sodium fluorosilicate to anhydrous sodium silicate) could be negligible. For the indices of elastic modulus, compressive strength, and tensile strength, the shared characteristic, where the biggest effect is $C$ (the mass ratio of anhydrous sodium silicate to aggregate), followed by $A$ (the proportion of fine powder to aggregates), $D$ (the mass ratio of sodium fluorosilicate to anhydrous sodium silicate), and $B$ (the proportion of barite sand to coarse aggregate), is seen.

(4) Regression equations of the parameters including density, Young's modulus, compressive strength, and tensile strength were obtained by using orthogonal polynomial regression analysis. The experiments and 


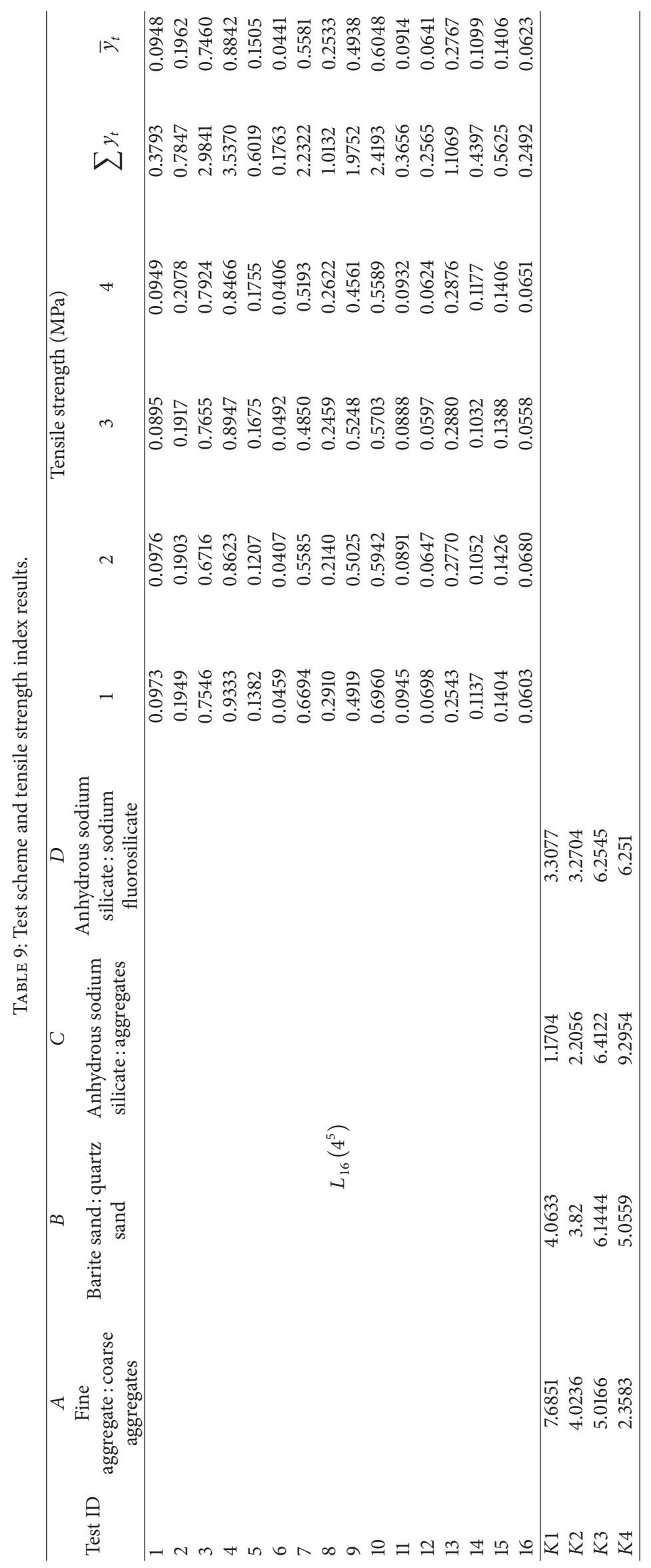


TABLE 10: Orthogonal polynomial regression analysis of variance: tensile strength index.

\begin{tabular}{|c|c|c|c|c|c|c|c|}
\hline The source of variance & $\begin{array}{l}\text { Square and } \\
\text { variation }\end{array}$ & $\begin{array}{l}\text { Degree of } \\
\text { freedom }\end{array}$ & $\begin{array}{l}\text { Variance } \\
\text { estimate }\end{array}$ & $F$-value & $F_{0.05}(1,51)$ & $\begin{array}{c}\text { Level of } \\
\text { significance }\end{array}$ & Remarks \\
\hline$b_{1 a}$ & $7.0194 \times 10^{-1}$ & 1 & $7.0194 \times 10^{-1}$ & 438.56 & 4 & $* * * *$ & $A, 1$-order term \\
\hline$b_{2 a}$ & $1.5725 \times 10^{-2}$ & 1 & $1.5725 \times 10^{-2}$ & 9.82 & & * & $A, 2$-order term \\
\hline$b_{3 a}$ & $2.1558 \times 10^{-1}$ & 1 & $2.1558 \times 10^{-1}$ & 134.69 & & $* * *$ & $A, 3$-order term \\
\hline$b_{1 b}$ & $8.7854 \times 10^{-2}$ & 1 & $8.7854 \times 10^{-2}$ & 54.89 & & $* *$ & $B, 1$-order term \\
\hline$b_{2 b}$ & $1.1162 \times 10^{-2}$ & 1 & $1.1162 \times 10^{-2}$ & 6.97 & & $*$ & $B, 2$-order term \\
\hline$b_{3 b}$ & $1.1177 \times 10^{-1}$ & 1 & $1.1177 \times 10^{-1}$ & 69.83 & & $* *$ & $B, 3$-order term \\
\hline$b_{1 c}$ & $2.5528 \times 10^{0}$ & 1 & $2.5528 \times 10^{0}$ & 1594.97 & & $* * * * *$ & $C, 1$-order term \\
\hline$b_{2 c}$ & $5.3361 \times 10^{-2}$ & 1 & $5.3361 \times 10^{-2}$ & 33.34 & & $* *$ & $C, 2$-order term \\
\hline$b_{3 c}$ & $6.3135 \times 10^{-2}$ & 1 & $6.3135 \times 10^{-2}$ & 39.45 & & $* *$ & $C, 3$-order term \\
\hline$b_{1 d}$ & $4.3616 \times 10^{-1}$ & 1 & $4.3616 \times 10^{-1}$ & 272.50 & & $* * * *$ & $D, 1$-order term \\
\hline$b_{2 d}$ & $1.7851 \times 10^{-5}$ & 1 & $1.7851 \times 10^{-5}$ & 0.01 & & & $D, 2$-order term \\
\hline$b_{3 d}$ & $1.1284 \times 10^{-1}$ & 1 & $1.1284 \times 10^{-1}$ & 70.50 & & $* *$ & $D, 3$-order term \\
\hline Error & $8.1629 \times 10^{-2}$ & 51 & $1.6006 \times 10^{-3}$ & & & & \\
\hline
\end{tabular}

TABLE 11: The range of mechanical parameters of objective and prototype materials.

\begin{tabular}{lcccc}
\hline & Density $\left(\mathrm{g} / \mathrm{cm}^{3}\right)$ & $\begin{array}{c}\text { Young's } \\
\text { modulus }(\mathrm{GPa})\end{array}$ & $\begin{array}{c}\text { Compressive } \\
\text { strength }(\mathrm{MPa})\end{array}$ & Tensile strength $(\mathrm{MPa})$ \\
\hline 1\# prototype material & $2.800 \sim 2.900$ & $20 \sim 35$ & $500 \sim 1000$ & $140 \sim 150$ \\
1\# objective material & $2.800 \sim 2.900$ & $0.20 \sim 0.35$ & $5 \sim 10$ & $1.4 \sim 1.5$ \\
1\# prototype material & $2.100 \sim 2.200$ & $3 \sim 4$ & $20 \sim 40$ & $10 \sim 20$ \\
2\# objective material & $2.100 \sim 2.200$ & $0.03 \sim 0.04$ & $0.2 \sim 0.4$ & $0.1 \sim 0.2$ \\
\hline
\end{tabular}

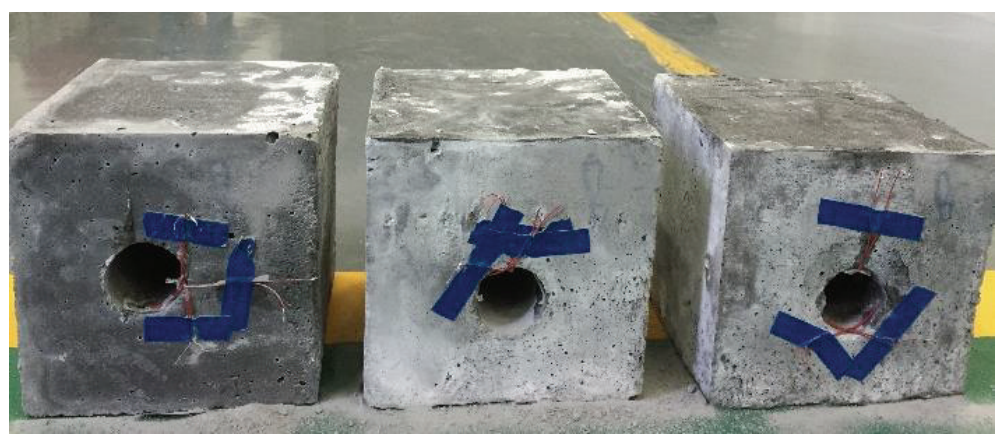

(a) Scaling tunnel model without reinforcement layer
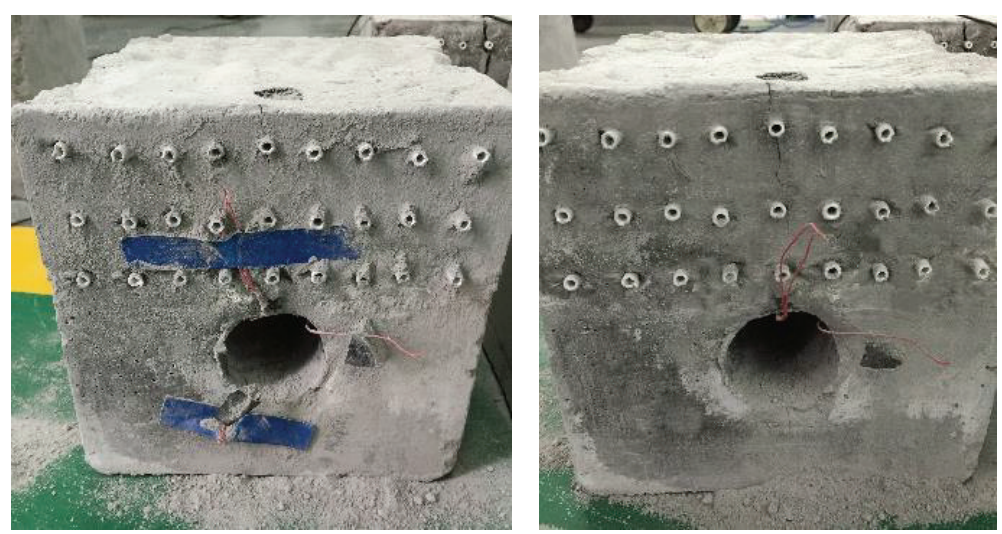

(b) Scaling tunnel model within reinforcement layers

FIGURE 13: Some specimens made of number 2 analogue material. 
TABLE 12: Raw material configurations and the measured mechanical indices of analogue materials.

\begin{tabular}{|c|c|c|c|c|c|c|c|}
\hline \multirow[b]{2}{*}{ Material ID } & \multicolumn{3}{|c|}{ Raw material configuration } & \multicolumn{4}{|c|}{ Measured mechanical index } \\
\hline & $\begin{array}{l}A \text { (the } \\
\text { proportion of } \\
\text { fine powder to } \\
\text { aggregates) }\end{array}$ & $\begin{array}{c}B \text { (the } \\
\text { proportion of } \\
\text { barite sand to } \\
\text { coarse } \\
\text { aggregate) }\end{array}$ & $\begin{array}{c}C \text { (the mass } \\
\text { ratio of } \\
\text { anhydrous } \\
\text { sodium silicate } \\
\text { to aggregate) }\end{array}$ & $\begin{array}{l}\text { Density } \\
\left(\mathrm{g} / \mathrm{cm}^{3}\right)\end{array}$ & $\begin{array}{c}\text { Young's } \\
\text { modulus } \\
(\mathrm{GPa})\end{array}$ & $\begin{array}{l}\text { Compressive strength } \\
\qquad(\mathrm{MPa})\end{array}$ & $\begin{array}{c}\text { Tensile strength } \\
(\mathrm{MPa})\end{array}$ \\
\hline $\begin{array}{l}\text { 1\# analogue } \\
\text { material }\end{array}$ & $30 \%$ & $50 \%$ & $3 \%$ & 2.855 & 0.286 & 6.058 & 1.469 \\
\hline $\begin{array}{l}2 \# \text { analogue } \\
\text { material }\end{array}$ & $65 \%$ & $00 \%$ & $3 \%$ & 2.181 & 0.035 & 0.315 & 0.180 \\
\hline
\end{tabular}

Note: the mass ratio of sodium fluorosilicate to anhydrous sodium silicate was $3: 4$ (this was the optimal value).

applications indicated that the properties of analogue materials were stable and predictable. It was easy to obtain objective material from the regression equations and trial test.

\section{Appendix}

See Tables 3-10.

\section{Competing Interests}

The authors declare that there is no conflict of interests arising from the work reported in, or the publication of, this paper.

\section{Authors' Contributions}

Songlin Yue, Yanyu Qiu, and Pengxian Fan conceived and designed the study. Songlin Yue, Pin Zhang, and Ning Zhang performed the experiments. Songlin Yue and Pengxian Fan wrote the paper. Yanyu Qiu, Pin Zhang, and Ning Zhang reviewed and edited the paper. All authors read and approved the paper.

\section{Acknowledgments}

The authors acknowledge the financial support from the Natural Science Foundation of China (Grants 51308543 and 51304219), the China Postdoctoral Science Foundation Funded Project (Grant 2015T81074), and the Open Fund Project of State Key Laboratory of Coal Resources and Safe Mining, CUMT (Grant 14KF02).

\section{References}

[1] J. Ubilla, T. Abdoun, and R. Dobry, "Centrifuge scaling laws of pile response to lateral spreading," International Journal of Physical Modelling in Geotechnics, vol. 11, no. 1, pp. 2-22, 2011.

[2] R. T. Klinkvort, O. Hededal, and S. M. Springman, "Scaling issues in centrifuge modelling of monopiles," International Journal of Physical Modelling in Geotechnics, vol. 13, no. 2, pp. 38-49, 2013.

[3] M. He, "Physical modeling of an underground roadway excavation in geologically $45 \circ$ inclined rock using infrared thermography," Engineering Geology, vol. 121, no. 3-4, pp. 165-176, 2011.
[4] D. P. David and C. F. Raymond, Fundamentals of Structural Geology, Cambridge University Press, Cambridge, UK, 2005.

[5] J. Garnier, C. Gaudin, S. Springman et al., "Catalogue of scaling laws and similitude questions in geotechnical centrifuge modelling," International Journal of Physical Modelling in Geotechnics, vol. 7, no. 3, pp. 01-23, 2007.

[6] P. Fan, M. Wang, H. Xing, K. Jiang, and Z. Li, “Time-dependent problems of deformation and failure in geo-mechanical model tests," Chinese Journal of Rock Mechanics \& Engineering, vol. 33, no. 9, pp. 1843-1851, 2014.

[7] F. Pengxian, W. Mingyang, and F. Xiang, "The boundary conditions of model test for deep-buried engineering and its simulation methods," Journal of Mining \& Safety Engineering, vol. 33, no. 1, pp. 146-151, 2016.

[8] E. Fumagalli, Statical and Geomechanical Models, chapter 2, Springer, New York, NY, USA, 1973.

[9] B. Stimpson, "Modelling materials for engineering rock mechanics," International Journal of Rock Mechanics and Mining Sciences \& Geomechanics Abstracts, vol. 7, no. 1, pp. 77-121, 1970.

[10] W. Burgert and H. Lippmann, "Models of translatory rock bursting in coal," International Journal of Rock Mechanics and Mining Sciences \& Geomechanics Abstracts, vol. 18, no. 4, pp. 285-294, 1981.

[11] B. Indraratna, "Development and applications of a synthetic material to simulate soft sedimentary rocks," Geotechnique, vol. 40, no. 2, pp. 189-200, 1990.

[12] F. P. Glushinkhin, G. N. Kutsnetsov, M. F. Shklyarsky et al., Modeling in Geo-Mechanics, Nedra, Moscow, Russia, 1991.

[13] P. Dykeman and A. J. Valsangkar, "Model studies of socketed caissons in soft rock," Canadian Geotechnical Journal, vol. 33, no. 5, pp. 747-759, 1996.

[14] Z. Li, D. Lu, H. Nakayama, H. Hosomi, and J. Sun, "Development and application of new technology for 3D geomechanics model test of large underground houses," Chinese Journal of Rock Mechanics and Engineering, vol. 22, no. 9, pp. 1430-1436, 2003.

[15] L. Dunham, A. J. Valsangkar, and A. B. Schriver, "Centrifuge modeling of rigid square footings on weak jointed rock," Geotechnical Testing Journal, vol. 28, no. 2, pp. 133-143, 2005.

[16] L.-W. Chen and S.-W. Bai, "Proportioning test study on similar material of rockburst tendency of brittle rockmass," Rock and Soil Mechanics, vol. 27, pp. 1050-1054, 2006.

[17] Q.-Y. Zhang, S.-C. Li, and X.-H. Guo, "Research and development of new typed cementitious geotechnical similar material 
for iron crystal sand and its application," Rock and Soil Mechanics, vol. 29, no. 8, pp. 2126-2130, 2008.

[18] B. Imre, B. Wildhaber, and S. M. Springman, "A physical analogue material to simulate sturzstroms," International Journal of Physical Modelling in Geotechnics, vol. 11, no. 2, pp. 69-86, 2011.

[19] P. Lin, X. Liu, W. Zhou, R. Wang, and S. Wang, "Cracking, stability and slope reinforcement analysis relating to the Jinping dam based on a geomechanical model test," Arabian Journal of Geosciences, vol. 8, no. 7, pp. 4393-4410, 2014.

[20] Z. Hui, M. Fanzhen, Z. Chuanqing, L. Jingjing, and X. Rongchao, "Review and status of research on physical simulation test for rockburst," Journal of Rock Mechanics \& Geotechnical Engineering, vol. 34, no. 5, pp. 915-923, 2015.

[21] Z. Weishen, L. Yong, L. Shucai, W. Shugang, and Z. Qianbing, "Quasi-three-dimensional physical model tests on a cavern complex under high in-situ stresses," International Journal of Rock Mechanics and Mining Sciences, vol. 48, no. 2, pp. 199-209, 2011.

[22] W. S. Zhu, Q. B. Zhang, H. H. Zhu et al., "Large-scale geomechanical model testing of an underground cavern group in a true three-dimensional (3-D) stress state," Canadian Geotechnical Journal, vol. 47, no. 9, pp. 935-946, 2010.

[23] P. Fan, H. Xing, L. Ma et al., "Bulk density adjustment of resin-based equivalent material for geomechanical model test," Advances in Materials Science and Engineering, vol. 2015, Article ID 363869, 8 pages, 2015.

[24] X.-G. Chen, Q.-Y. Zhang, Y. Wang, S.-C. Li, and H.-P. Wang, "In situ observation and model test on zonal disintegration in deep tunnels," Journal of Testing and Evaluation, vol. 41, no. 6, pp. 1-11, 2013.

[25] D. E. Gill, R. Corthésy, and M. H. Leite, "Determining the minimal number of specimens for laboratory testing of rock properties," Engineering Geology, vol. 78, no. 1-2, pp. 29-51, 2005.

[26] Q. Wang and L. Wu, "Determination of elastic modulus, tensile strength and fracture toughness of brittle rocks by using flattened brazilian disk specimen-part II: experimental results," Chinese Journal of Rock Mechanics and Engineering, vol. 23, no. 2, pp. 199-204, 2004.

[27] W. Ren, C. Guo, Z. Peng, and Y. Wang, "Model experimental research on deformation and subsidence characteristics of ground and wall rock due to mining under thick overlying terrane," International Journal of Rock Mechanics \& Mining Sciences, vol. 47, no. 4, pp. 614-624, 2010. 

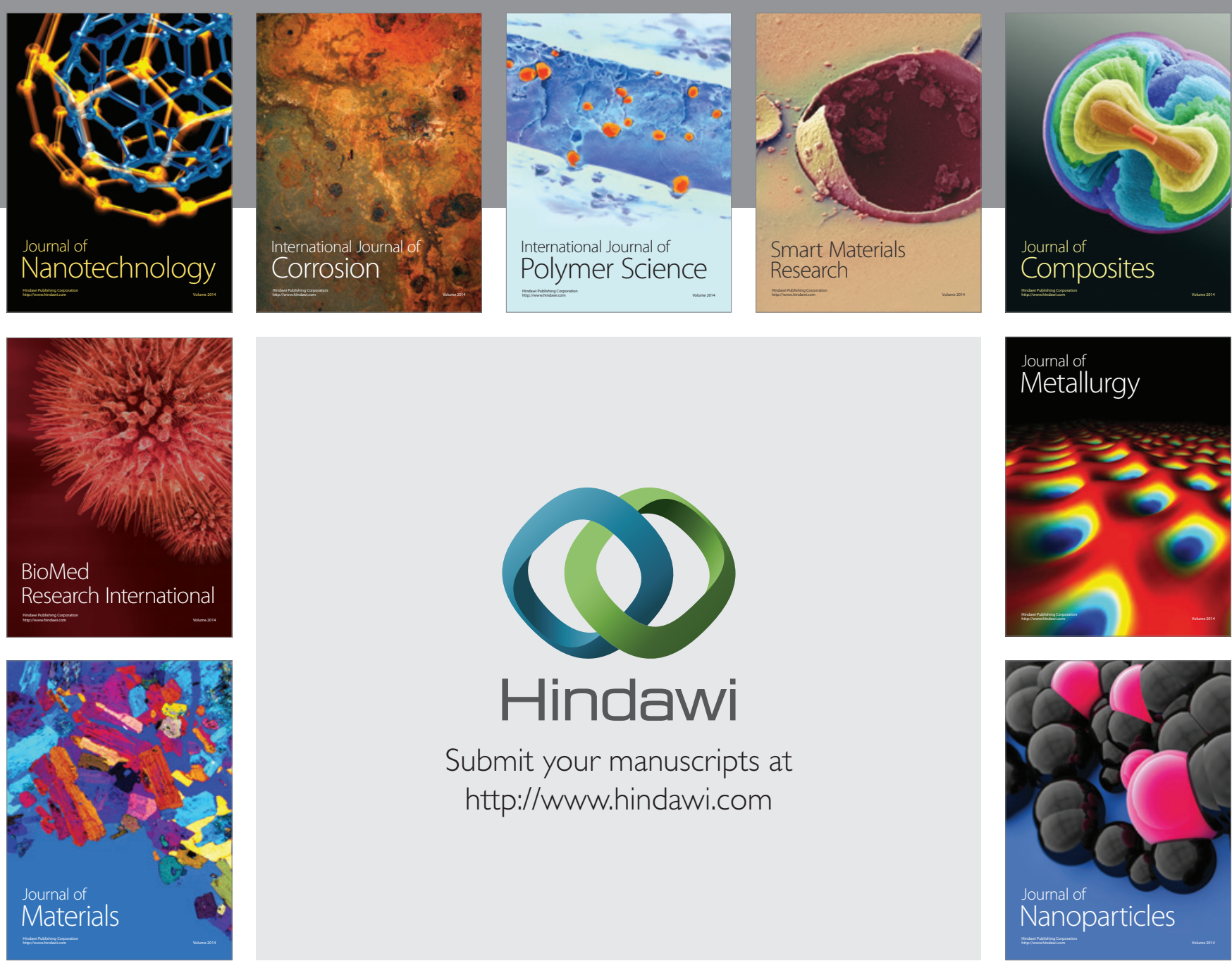

\section{Hindawi}

Submit your manuscripts at

http://www.hindawi.com

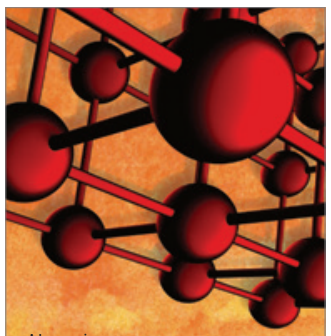

Materials Science and Engineering
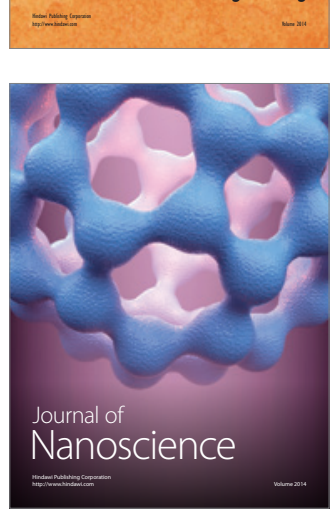
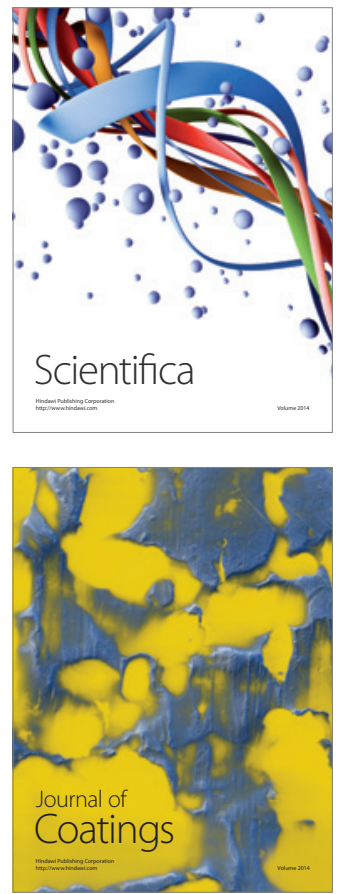
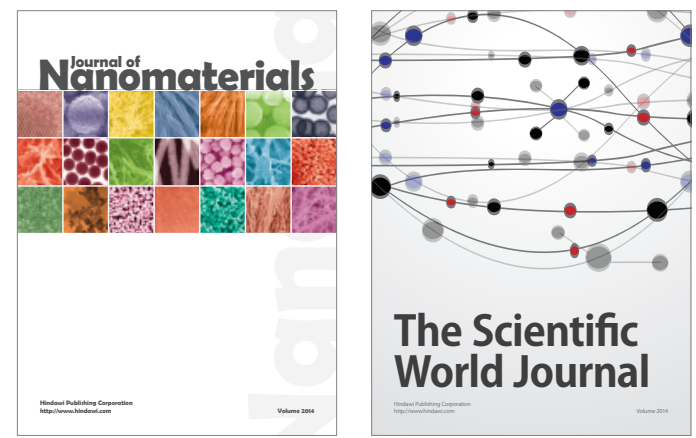

The Scientific World Journal
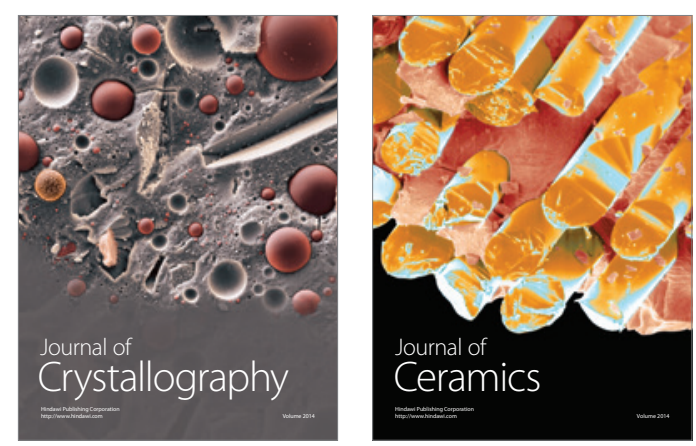
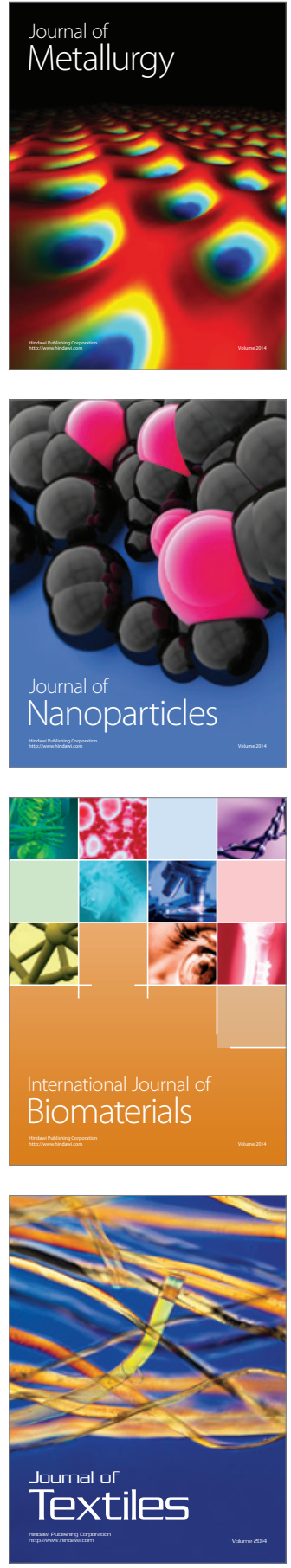